\title{
ESTUDOS DE ESTABILIDADE ACELERADA DE EMULSÕES MANIPULADOS CONTENDO O ANTIVIRAL ACICLOVIR
}

\section{ACELERATED STABILITY STUDY OF MANIPULATED EMULSIONS WITH ANTIVIRAL ACICLOVIR}

\author{
Monique Carniel BELTRAMI ${ }^{1}$, Rosana BASSO ${ }^{1}$, Marcos Antônio Segatto SILVA ${ }^{1}$, Simone \\ CARDOSO ${ }^{1}$, Hellen Karine STULZER ${ }^{1,2}$.
}

\begin{abstract}
${ }^{1}$ Laboratório de Controle de Qualidade, Departamento de Ciências Farmacêuticas, Centro de Ciências da Saúde, Universidade Federal de Santa Catarina, Campus Trindade, 88040900, Florianópolis, SC, Brasil.

${ }^{2}$ Laboratório de Controle de Qualidade, Departamento de Ciências Farmacêuticas, Universidade Estadual de Ponta Grossa, Campus Uvaranas, 84010-919, Ponta Grossa, PR, Brasil.

RECEBIDO: 04/04/08 ACEITE: 11/05/08
\end{abstract}

\begin{abstract}
RESUMO:
A escolha adequada do veículo à qual a substância ativa será incorporada é de fundamental importância para a estabilidade e absorção da mesma. Além disso, tem por finalidade avaliar o comportamento dos medicamentos em função do tempo e a influência de uma variedade de condições e fatores.

No presente estudo foi avaliada a influência de três distintas bases: aniônica (Lanette $\AA$ ), não-iônica (Cosmowax $\left.{ }^{\circledR}\right)$ e iônica $(\mathrm{A} / \mathrm{E} 165 \mathrm{M} \AA)$ na estabilidade de emulsões contendo aciclovir, comparando-as entre si e com o medicamento referência Zovirax® e genérico (Medley). Os parâmentos avaliados foram, propriedades físicas, $\mathrm{pH}$, separação de fases e doseamento.

Os estudos de estabilidade indicaram que as três amostras manipuladas apresentaram concentração inicial de aciclovir e pH abaixo do preconizado. A base Cosmowax ${ }^{\circledR}$ apresentou coloração amarelada após 28 dias de análise e aspecto grumoso. A mesma também indicou contaminação microbiana e maior degradação quando submetida a valores de temperatura e umidade elevados, possuindo assim, um menor prazo de validade quando comparado às demais formulações manipuladas.
\end{abstract}

Palavras - chave: emulsão, estabilidade, aciclovir

\begin{abstract}
:
The correct choice of the vehicle where the active substance will be incorporated is of major importance for the cream stability and absorption. Moreover, it has for purpose to evaluate the behavior of medicines in function of the time and the influence of a variety of conditions and factors. At the present work, the influence of three different bases was evaluated: anionic (Lanette $\AA$ ), non-ionic (Cosmowax $®$ ) and ionic $(\mathrm{A} / \mathrm{E} 165 \mathrm{M} 囚)$ according to the stability of emulsions with acyclovir, comparing them among each other and with the reference medicine Zovirax ${ }^{\circledR}$ and the generic (Medley). The parameters evaluated were: physical properties, $\mathrm{pH}$, phase separation and dosage. The stability studies shown that the three compounded preparation samples had initial acyclovir concentration and $\mathrm{pH}$ above the recommended value. Cosmowax ${ }^{\circledR}$ turned yellow 28 days after the analysis and had a lumpy aspect. It also indicated microbiological contamination and higher degradation after exposed to high temperature and humidity, indicating a shorter shelf life when compared to the other pharmaceutical compounded preparations.

Keywords: emulsion, stability, acyclovir.
\end{abstract}




\section{INTRODUÇÃO}

O grande número de substâncias ativas disponíveis, com diferentes propriedades físicoquímicas e farmacodinâmicas faz com que, cada vez mais, tenha-se que conhecer os veículos compatíveis com a manutenção da estabilidade da formulação final, para que não ocorra comprometimento da ação do produto. No mercado existem vários veículos que podem ser usados na preparação de emulsões, formulações popularmente conhecidas como loções ou cremes [1]. A escolha adequada do veículo à qual a substância ativa será incorporada é de fundamental importância para a estabilidade e absorção desta e, conseqüentemente, para a eficácia do produto final [2].

Emulsões são preparações farmacêuticas obtidas pela dispersão de duas fases líquidas imiscíveis. De acordo com a hidrofilia ou a lipofilia da fase dispersante, estes sistemas são classificados em óleo-em-água (O/A) ou em água-em-óleo (A/O) [3]. As preparações emulsionadas são termodinamicamente instáveis e, com o tempo podem apresentar eventual separação de fases. A estabilidade é definida pela extensão de tempo no qual o produto mantém, dentro de limites especificados pelo período de armazenamento e uso, as mesmas propriedades que possuía no momento da sua fabricação $[1,4]$.

Os estudos na fase de pré-formulação incluem a estabilidade no estado sólido do fármaco isolado e a estabilidade na presença dos excipientes. Estas etapas são realizadas para reduzir ou prevenir a ocorrência de deterioração devido à hidrólise, oxidação e outros processos $[2,5]$. O estudo da estabilidade da formulação tem por finalidade avaliar o comportamento dos medicamentos em função do tempo e a influência de uma variedade de condições e fatores, sendo levado em consideração tanto o fármaco, quanto a mistura de excipientes ou veículos utilizados, assim como a interação entre ambos, face às condições às quais estão submetidos [2,6].

A literatura descreve que o aciclovir apresenta excelente estabilidade em formas farmacêuticas sólidas, entretanto, este sofre degradação quando exposto em solução aquosa, sendo o principal produto de degradação a guanina [7].

Dessa forma, o presente estudo propõe a avaliação da estabilidade acelerada em curto prazo da forma farmacêutica creme, contendo aciclovir incorporado em três distintas bases: aniônica (Lanette $®)$, não-iônica (Cosmowax®) e iônica $(A / E 165 \mathrm{M} 囚)$, bem como a comparação com o medicamento de referência Zovirax® e genérico (Medley) deste fármaco.

\section{MATERIAL E MÉTODOS}

\subsection{Material}

A substância química de referência (SQR) utilizada como padrão secundário, foi aciclovir com teor declarado de 99,9 \% (lote PRX2006018, China). Os reagentes utilizados, álcool etílico, ácido clorídrico e cloreto de sódio apresentavam grau analítico.

As três formulações de aciclovir creme manipuladas em diferentes bases: aniônica (Lanette $\AA)$, não-iônica (Cosmowax®) e iônica $(A / E$ 165M®) estão descritas na Tabela 1.

As emulsões foram preparadas da maneira habitual, fundindo separadamente a fase $A$ (aquosa) e a fase $\mathrm{O}$ (oleosa) na temperatura de $75^{\circ} \mathrm{C}$ em banho-maria, sob agitação manual constante. Após as duas fases atingirem $40^{\circ} \mathrm{C}$, a fase aquosa foi vertida sob a fase oleosa. Manteve-se agitação em banho-maria por alguns minutos e em seguida, o produto foi retirado do banho e a agitação mantida até o resfriamento. A emulsão foi deixada em repouso por algumas horas em temperatura ambiente, sendo em seguida o aciclovir incorporado. As mesmas foram preparadas de acordo com o Manual de boas praticas de Fabricação [8]. 
TABELA 1. Formulações desenvolvidas.

\begin{tabular}{cccc}
\hline Descrição & $\begin{array}{c}\text { Lanette }{ }^{\circledR} \\
\text { (aniônica) }\end{array}$ & $\begin{array}{c}\text { Cosmowax }{ }^{\circledR} \\
\text { (não-iônica) }\end{array}$ & $\begin{array}{c}\text { A/E165M }{ }^{\circledR} \\
\text { (iônica) }\end{array}$ \\
\hline Vaselina líquida & $8,0 \%$ & $5,0 \%$ & $1,0 \%$ \\
Água destilada & $1,0 \%$ qsp & $1,0 \%$ qsp & $1,0 \%$ qsp \\
Phenova & $0,3 \%$ & $0,3 \%$ & $0,3 \%$ \\
Propilenoglicol & $7,0 \%$ & $5,0 \%$ & $5,0 \%$ \\
Cosmowax ${ }^{\circ}$ & ----- & $14,0 \%$ & ---- \\
Lanete ${ }^{2}$ & $13,0 \%$ & ----- & ----- \\
Cera A/E165M & ----- & ---- & $14,0 \%$ \\
Silicone super & ----- & $1,0 \%$ & $1,0 \%$ \\
volátil & & $0,02 \%$ & $0,05 \%$ \\
BHT & $0,1 \%$ & $0,2 \%$ & $0,2 \%$ \\
Nipagim & $0,2 \%$ & $0,1 \%$ & $0,1 \%$ \\
Nipazol & $0,1 \%$ & & \\
\hline
\end{tabular}

\subsection{Estabilidade acelerada}

O estudo de estabilidade acelerada em curto prazo foi realizado durante o período de 28 dias, com análise do teor de fármaco nos tempos zero (dia em que as amostras magistrais foram manipuladas), 7, 14, 21 e 28 dias, nas condições de temperatura e umidade ambiente ( $25{ }^{\circ} \mathrm{C} \pm 2{ }^{\circ} \mathrm{C}, 60 \%$ UR $5 \%$ UR) e câmara climática $\left(40{ }^{\circ} \mathrm{C} 2{ }^{\circ} \mathrm{C}, 75 \%\right.$ UR $5 \%$ UR). $\mathrm{O}$ teor do fármaco nas formulações foi avaliado por espectrofotometria de absorção na região ultravioleta. Os cremes referência e genérico foram submetidos apenas à câmara climática, nas mesmas condições.

\subsubsection{Avaliação das propriedades físicas}

As características organolépticas das amostras, tais como cor, odor, aparência, integridade, brilho, firmeza e coesividade foram determinadas por comparação com as especificações obtidas em literatura e com o medicamento referência.

2.2.2 Análise de $\mathrm{pH}$ - potencial hidrogeniônico

A determinação deste parâmetro foi avaliada em um pHmetro digital QUIMIS, previamente calibrado. 


\subsubsection{Separação de Fases}

Uma alíquota correspondente a $0,5 \mathrm{~g}$ da amostra foi transferida para um eppendorf e centrifugada a $4500 \mathrm{rpm}$ durante 15 minutos em centrifuga SIGMA. Após esse tempo, as amostras forma retiradas para análise, verificando-se a instabilidades através de separação de fases.

\subsubsection{Doseamento}

Analiticamente foi pesado uma quantidade do creme que correspondesse a aproximadamente $7,5 \mathrm{mg}$ de aciclovir. À amostra foi adicionado $50 \mathrm{~mL}$ de acido sulfúrico 0,5 M. Em seguida foi realizada a extração com $50 \mathrm{~mL}$ de acetato de etila, após a separação coletou-se a camada aquosa inferior mais límpida. Descartou-se a primeira alíquota e a volume correspondente a $10 \mathrm{~mL}$ desse filtrado, adicionou-se água destilada suficiente para produzir $50 \mathrm{~mL}$ de solução. Mediu-se a absorbância da solução resultante em espectrofotômetro UV/ Vis CARY BIO em $255 \mathrm{~nm}$ [9].

\subsubsection{Ensaio Microbiológico}

A análise microbiológica foi realizada com as três bases manipuladas (Cosmowax $®$ não-iônica, Lanette $\AA$ - aniônica, $A / E 165 \mathrm{M} 囚$ - iônica) e realizadas em tempo Zero e após 28 dias, para amostras que estavam armazenadas em temperatura ambiente e câmara climática. Os procedimentos foram realizados em triplicata para as diluições $10^{-1}$ e $10^{-4}$, utilizando meios para bactérias e fungos conforme metodologia descrita na Farmacopéia Brasileira [10].

\section{RESULTADOS E DISCUSSÃO}

\subsection{Avaliação das propriedades físicas}

A propriedade que as emulsões possuem de manter, de forma inalterada, as características que apresentavam, após a fabricação, como: cor, odor, aparência, integridade, brilho, firmeza e coesividade são consideradas propriedades físicas [5].

De acordo com os dados obtidos, observa-se que todas as formulações desenvolvidas mantiveram as características originais até o $21^{\circ}$ dia de ensaio, em ambas as condições analisadas.

Em relação aos parâmetros que sofreram alterações, ressalta-se a cor e a aparência para a base Cosmowax ${ }^{\circ}$, sendo que a mesma apresentou uma coloração amarelada em ambas às condições de análise e aspecto grumoso em câmara climática. Na mesma condição foi observado perda de brilho e firmeza.

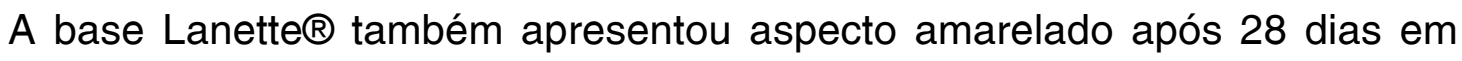
câmara climática. Para a base $A E 165 \mathrm{M} \circledast$, creme Zovirax $\AA$ e Genérico contendo aciclovir, as características avaliadas permaneceram constantes durante todo o período de ensaio. Dados expostos nas Tabelas 2, 3, 4 e 5. 
TABELA 2. Amostra nas condições de t emperatura ambiente e câmara climática nos tempos Zero e 28 dias.

\begin{tabular}{lllll}
\hline \multirow{2}{*}{$\begin{array}{c}\text { Cosmowax } \\
\text { (não-iônica) }\end{array}$} & \multirow{2}{*}{ Temp. Amb. } & Cam. Clima. & Temp. Amb. & Cam. Clima. \\
\hline Cor & Branco & Branco. & Amarelo & Amarelo \\
Odor & Característico & Característico & Característico & Característico \\
Aparência & Homogêneo & Homogêneo & Homogêneo & Grumoso \\
Integridade & Sim & Sim & Sim & Sim \\
Brilho & XX & XX & XX & X \\
Firmeza & XXX & XXX & XXX & XX \\
Coesividade & XXX & XXX & XXX & XXX \\
\hline
\end{tabular}

Nota: XXX - Ótimo; XX-Regular; X-Ruim.

TABELA 3. Amostra nas condições de t emperatura ambiente e câmara climática nos tempos Zero e 28 dias.

\begin{tabular}{lllll}
\hline \multicolumn{1}{c}{ Lanette ${ }^{\circledR}$} & \multicolumn{2}{c}{ Zero } \\
(aniônica) & Temp. Amb. & Cam. Clima. & Temp. Amb. & \multicolumn{1}{c}{ Cam. Clima. } \\
\hline Cor & Branco & Branco & Branco & Amarelado \\
Odor & Característico & Característico & Característico & Característico \\
Aparência & Homogêneo & Homogêneo & Homogêneo & Homogêneo \\
Integridade & Sim & Sim & Sim & Sim \\
Brilho & XX & XX & XX & XX \\
Firmeza & X & X & X & X \\
Coesividade & XX & XX & XX & XX \\
\hline
\end{tabular}

Nota: XXX - Otimo; XX-Regular; X - Ruim.

TABELA 4. Amostra nas cond ições de $t$ emperatura ambiente e câmara climática nos tempos Zero e 28 dias.

\begin{tabular}{lllll}
\hline \multicolumn{1}{c}{ A/E165M® } & \multicolumn{2}{c}{ Zero } \\
\multicolumn{1}{c}{ (iônica) } & Temp. Amb. & Cam. Clima. & Temp. Amb. & Cam. Clima. \\
\hline Cor & Branco & Branco & Branco & Branco \\
Odor & Característico & Característico & Característico & Característico \\
Aparência & Homogêneo & Homogêneo & Homogêneo & Homogêneo \\
Integridade & Sim & Sim & Sim & Sim \\
Brilho & XXX & $X X X$ & $X X X$ & XXX \\
Firmeza & $X$ & $X$ & $X$ & $X$ \\
Coesividade & $X X$ & $X X$ & $X X$ & $X X$ \\
\hline
\end{tabular}

Nota: XXX - Ótimo; XX-Regular; X - Ruim. 
TABELA 5. Amostras submetidas à câmara climática nos tempos Zero e 28 dias.

\begin{tabular}{lllll}
\hline \multicolumn{1}{c}{$\begin{array}{c}\text { Câmara } \\
\text { Climática }\end{array}$} & \multicolumn{2}{c}{ Referência (Zovirax®) } & \multicolumn{2}{c}{ Genérico (Medley) } \\
& \multicolumn{1}{c}{ Zero } & \multicolumn{1}{c}{ 28 dias } & \multicolumn{1}{c}{ Zero } & \multicolumn{1}{c}{28 dias } \\
\hline Cor & Branco & Branco & Branco & Branco \\
Odor & Característico & Característico & Característico & Característico \\
Aparência & Homogêneo & Homogêneo & Homogêneo & Homogêneo \\
Integridade & Sim & Sim & Sim & Sim \\
Brilho & $X X X$ & $X X X$ & $X X$ & $X X$ \\
Firmeza & $X X$ & $X X$ & $X X$ & $X X$ \\
Coesividade & $X X$ & $X X$ & $X X$ & $X X$ \\
\hline
\end{tabular}

Nota: XXX - Ótimo; XX-Regular; X-Ruim.

A instabilidade das formulações farmacêuticas pode ser detectada em alguns casos por uma mudança na aparência física, na cor, odor, gosto ou na textura, enquanto em outros casos, podem ocorrer alterações químicas que não são evidentes e que só podem ser verificadas por análise química [2,11].

Nas amostras submetidas à câmara climática a instabilidade pode ser detectada visualmente conforme Figura 1.

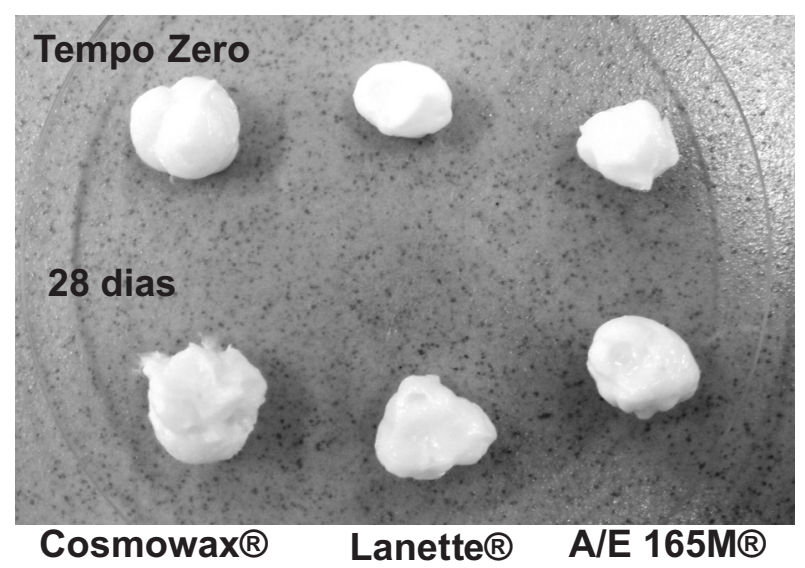

FIGURA 1. Imagem das diferentes formulações quando submetidas à câmara climática no tempo Zero e após o período de 28 dias.

\subsection{Análise de $\mathrm{pH}$}

Os valores de $\mathrm{pH}$ estão relacionados com alterações químicas, que podem influenciar diretamente a estabilidade da formulação, sendo que esses em formulações tópicas devem ter valores entre 5,5 e 7,0 pH cutâneo [12]. Conforme as tabelas 6 e 7, nas três amostras manipuladas, os pH's sofreram um aumento como passar do tempo, em ambas as condições analisadas.

Os medicamentos referência (Zovirax®) e genérico (Medley) permaneceram com os valores de pH's próximos ao ideal durante todo o período de análise. 
TABELA 6. Resultados obtidos nos ensaios de pH em temperatura ambiente

\begin{tabular}{lrrr}
\hline Tempo & Lanette® & Cosmowax® & AVE 165M® \\
\hline Zero & 7,00 & 7,05 & 7,40 \\
7 & 10,75 & 10,82 & 10,55 \\
14 & 10,40 & 10,83 & 10,66 \\
21 & 10,47 & 11,02 & 10,75 \\
28 & 11,12 & 11,09 & 11,01 \\
\hline
\end{tabular}

TABELA 7. Resultados obtidos nos ensaios de pH em câmara climática

\begin{tabular}{lrrrrr}
\hline Tempo & Lanette® & Cosmowax® & AVE 165M ® & Referência & Genérico \\
\hline Zero & 7,00 & 7,05 & 7,40 & 6,76 & 6,95 \\
7 & 10,25 & 10,90 & 10,71 & 6,84 & 7,00 \\
14 & 10,26 & 11,67 & 10,81 & 6,95 & 7,06 \\
21 & 10,75 & 11,68 & 11,06 & 7,33 & 7,15 \\
28 & 11,37 & 11,69 & 11,05 & 7,40 & 7,26 \\
\hline
\end{tabular}

\subsection{Separação de fases}

Este ensaio foi realizado através de centrifugação, o qual fornece informações rápidas sobre sinais de instabilidade do sistema, como a sedimentação e a separação de fases. Todas as amostras armazenadas em temperatura ambiente e em câmara climática apresentaram-se estáveis em relação a este parâmetro [13].

\subsection{Teor de aciclovir}

A diminuição da concentração de fármaco em cremes está diretamente relacionada à não adequação da base, por seu pH, cargas iônicas opostas, ausência de um sistema antioxidante-seqüestrante adequado, ausência de sistema conservante de amplo espectro, problemas na incorporação do ativo, devido a dificuldades de solubilidade e interação com outros componentes [14].

As análises indicaram uma diminuição na concentração de aciclovir (figuras 1a e 1b) com o decorrer do tempo para as três amostras manipuladas, nas duas condições de temperatura e umidade. A partir dos ensaios realizados no tempo Zero, observou-se que estas três bases apresentavam concentrações de fármaco inferior a faixa de $95-105 \%$ para a forma farmacêutica creme [9]. 
Estes resultados apontam que a incorporação do fármaco nas bases manipuladas não alcançou os níveis de aceitabilidade desejada. Provavelmente devido às características da própria base aliada aos demais constituintes da formulação. Estes fatores em conjunto não proporcionarem uma adequada distribuição do principio ativo.

Estudos realizados por Trottet e colaboradores [15] apontaram que formulações na forma farmacêutica de creme contendo aciclovir com $40 \%$ de propilenoglicol apresentam melhor permeabilidade e eficácia em relação a formulações contendo o mesmo excipiente em menor quantidade. As formulações manipuladas apresentaram baixa concentração deste componente, característica esta que pode ter influenciado em uma menor distribuição do fármaco na formulação.

Os medicamentos referência (Zovirax $囚$ ) e genérico (Medley) apresentaram concentrações praticamente inalteradas no decorrer das análises. De acordo com o formulário da bula destes medicamentos, estes possuem uma quantidade de propilenoglicol igual ou superior a $40 \%$.

A base contendo aciclovir que apresentou uma queda mais acentuada do fármaco foi a Cosmowax®, tanto na temperatura ambiente quanto para as amostras submetidas à câmara climática. Estes resultados condizem com o observado nos demais ensaios.
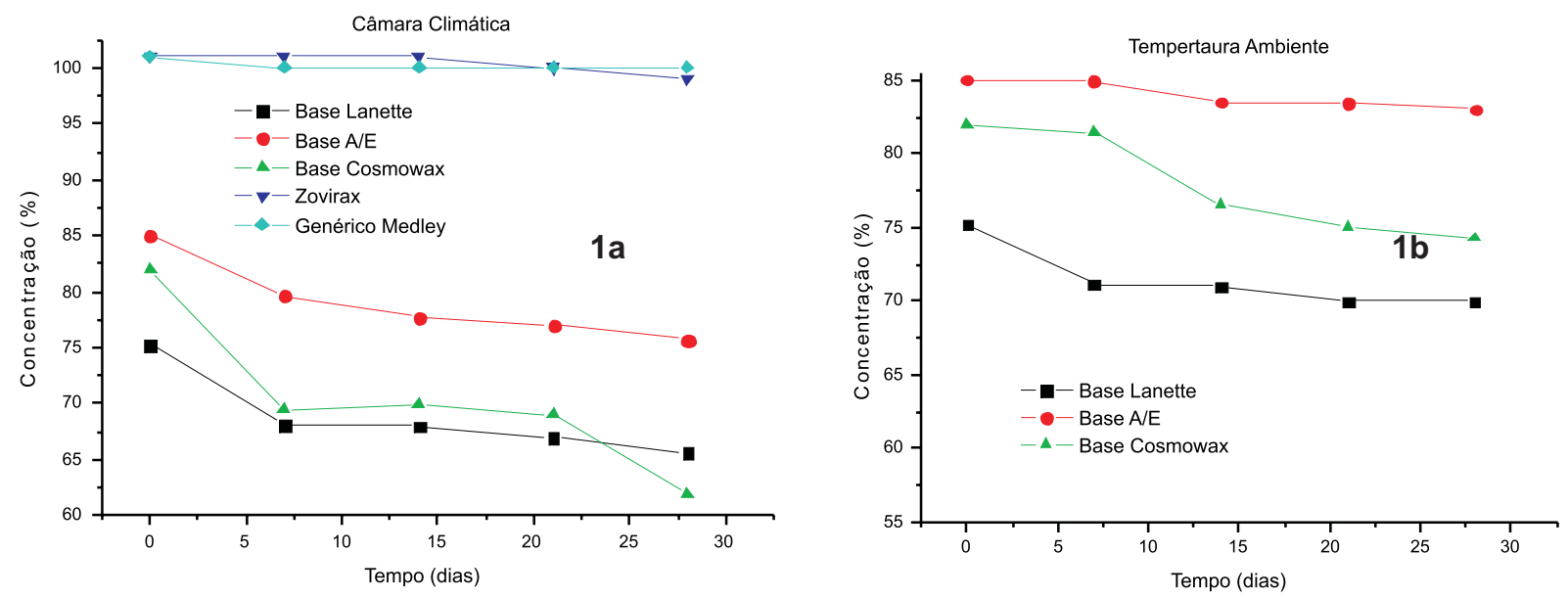

FIGURA 2. Doseamento do aciclovir em cremes manipulados, referência (Zovirax®) e genérico (Medley), submetidos à câmara climática (1a), e temperatura ambiente para os três cremes manipulados (1b).

O estudo cinético inicia-se com a medida da concentração do fármaco em intervalos determinados, sob um conjunto específico de condições que incluem temperatura, $\mathrm{pH}$, umidade, concentração iônica, intensidade da luz e concentração do fármaco. A temperatura afeta a estabilidade de um fármaco por meio do aumento da velocidade de reação, em média duas a três vezes para cada aumento de $10^{\circ} \mathrm{C}$ na temperatura. Para que as constantes de velocidade ou a velocidade de degradação possam ser usadas na formulação de preparações farmacêuticas é necessário avaliar o efeito da temperatura sobre a reação. Esta determinação permite prever a estabilidade do produto para uma temperatura normal em prateleira, a partir dos resultados obtidos nos ensaios de estabilidade acelerada [14].

A estabilidade e o prazo de validade baseiam-se na cinética de reação, isto é, o estudo da velocidade da interação química e o modo como essa velocidade é influenciada pelas condições ambientais a que o produto for submetido [5]. 
$\mathrm{Na}$ Tabela 8, os valores de $\left(\mathrm{r}^{2}\right)$ estão relacionados com a ordem da reação. Para todas as amostras manipuladas, a degradação do principio ativo seguiu uma cinética de segunda ordem. A constante de degradação a $2^{\circ} \mathrm{C}\left(\mathrm{K}_{25}\right)$ calculado apresentou diferentes valores, sendo o mais elevado, o encontrado para formulação manipulada em base Cosmowax $囚$, indicando assim que a mesma apresenta um menor prazo de validade $\left(T_{90}\right)$.

TABELA 8. Estudo cinético do comportamento das amostras manipuladas submetidos à temperatura ambiente.

\begin{tabular}{|c|c|c|c|c|}
\hline \multirow[b]{2}{*}{ Amostra } & \multicolumn{4}{|c|}{ Temperatura ambiente } \\
\hline & Ordem da reação & $\mathrm{K}_{25}$ & $r^{2}$ & ${ }^{\top} 90$ (dias) \\
\hline Base Lanette $\AA$ & Segunda ordem & $2,935 \times 10^{-6}$ & 0,7652 & 446,2 \\
\hline Base Cosmowax® & Segunda ordem & $1,032 \times 10^{-5}$ & 0,9834 & 140,6 \\
\hline Base A/E 165M® & Segunda ordem & $6,575 \times 10^{-6}$ & 0,8105 & 224,3 \\
\hline
\end{tabular}

Nota: $\mathrm{r}^{2}$; Coeficiente de correlação de Pearson, $\mathrm{K} \quad{ }_{25}$; Constante de velocidade de degradação à $25^{\circ} \mathrm{C}, \mathrm{T}_{90}$; Prazo de validade.

\subsection{Análise microbiológica}

Cargas microbianas elevadas podem também facilmente comprometer a estabilidade do produto. Conseqüências deste comprometimento estão associadas com a perda de eficácia terapêutica, seja por degradação do princípio ativo, seja por alteração de parâmetros físicoquímicos fundamentais para a sua atividade. A alteração dessas propriedades pode indiretamente afetar a ação terapêutica, comprometendo a disponibilidade do produto, assim como aceitação do mesmo pelo consumidor devido ao aparecimento de turbidez, mau cheiro e fermentações, além da produção de substâncias tóxicas [16].

As amostras manipuladas não apresentaram crescimento microbiano no tempo Zero. No entanto, observou-se crescimento de bactérias e fungos após 28 dias nas amostras que estavam em câmara climática, para a base Cosmowax®) (Figura 3). A contagem para bactérias apresentou mais de 1000 UFC. Também houve crescimento de fungos para a mesma amostra. Os limites de crescimento aceitáveis para bactérias e fungos são $1000 \mathrm{UFC/mL}$ e $100 \mathrm{UFC} /$ $\mathrm{mL}$, respectivamente. Os resultados apontaram níveis de contaminação acima do preconizado pela Farmacopéia Brasileira [10].

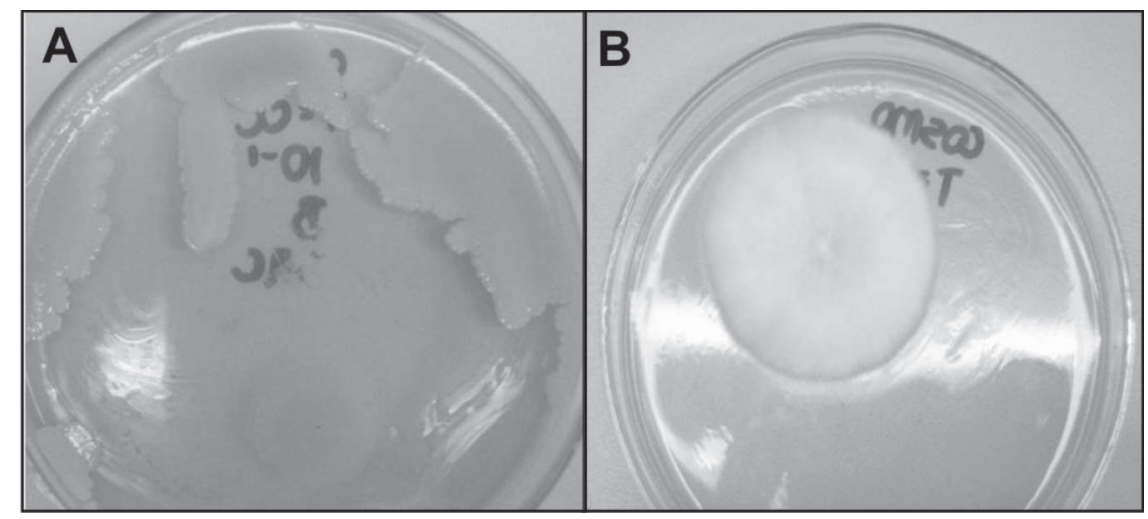

FIGURA 3. Crescimento de Bactérias (A) e Fungos (B) na análise do controle microbiológico. 


\section{CONCLUSÕES}

Através dos resultados expostos neste trabalho, pode-se constatar que as três amostras manipuladas apresentaram concentração inicial de aciclovir abaixo do preconizado. Os estudos de estabilidade indicaram que todas as amostras manipuladas apresentaram valores de $\mathrm{pH}$ diferentes do $\mathrm{pH}$ fisiológico, com o passar do tempo. Os dados apontam uma maior degradação para as amostras manipuladas em base Cosmowax®. A mesma base apresentou contaminação microbiana quando foi submetida a valores de temperatura e umidade elevados, possuindo assim, um menor prazo de validade quando comparado às demais formulações manipuladas.

Portanto, as formulações deveriam ser revistas quanto à composição de seus excipientes e um estudo de pré-formulação se faz necessário para que as formulações manipuladas sejam passíveis de intercambialidade com as formulações industrializadas.

\section{REFERÊNCIAS}

LEONARDI, G.R. Cosmetologia aplicada São Paulo: Editora, Medfarma, 2004.

ANSEL, H.C.; POPOVICH, N. G.; ALLEN, L. V. Farmacotécnica: formas farmacêuticas e sistemas de liberação de fármacos. 6. ed. São Paulo: Editora Premiere, 2000.

PRISTA, L. N.; ALVES, A. C.; MORGADO, R. Técnica Farmacêutica e Farmácia Galênica. 3. ed, v. 1, Fundação Calouste Gulbenkiam, Lisboa 1981.

THE UNITED STATES PHARMACOPEIA 26. ed., Rockville: United States Pharmacopeial Convention, 2006. Cd-room.

AULTON, M.E. "Delineamento de Formas Farmacêuticas". Artmed, Porto Alegre, Vol.1. 2005.

Grimm, W (1998) Drug Dev. Ind. Pharm. 24: 313-25

V.R. Sinha, Monika, A. Trehan, Manoj Kumar, S. Singh, and J.R. Bhinge. Stress Studies on Acyclovir. Journal of Chromatographic Science, Vol. 45, 2007. 319 p.

BRASIL. Ministério da Saúde. Agência Nacional de Vigilância (ANVISA) RE no 1450, de 11 de setembro de 2001, Certificado de Boas Práticas de Fabricação para Cosméticos. Lex: Disponível em: < http://www.anvisa.gov.br>.

BRITISH PHARMACOPEIA. London: Her Majesty's Stationery Office, 1999.vol.1.

FARMACOPÉIA BRASILEIRA. 4. ed. São Paulo: Atheneu, 1988. V. 1 Procedimentos técnicos aplicados a medicamentos.

Lachman, L., H.A. Lieberman \& J.L. Kaning ( 2001) "Teoria e Prática na Indústria Farmacêutica”. (Fundação Calouste), Lisboa, Vol. 1 e 2.

AZULAY, Rubem David; AZULAY, David Rubem. Dermatologia. 3. ed. Rio de Janeiro: Guanabara Koogan, 2004 
STULZER, H. K.; GONÇALVES, R.M.; FERREIRA, M.P. Loção Infantil para Assaduras. Cosmetics \& Toiletries. Vol 18, mar-abr. 2006. p. 90-93.

STULZER H.K.; SILVA M.A.S. Estudo de Estabilidade de Grânulos Revestidos e Comprimidos contendo Captopril. Acta Farm. Bonaerense 25 (4): 497-504 (2006).

L. Trottet, H. Owen, P. Holme, J. Heylings, I.P.Collin, A.P.Breen, M.N. Siyad, R.S.Nandra, A.F.Davis (2005). Are all aciclovir cream formulations bioequivalent?. International Journal of Pharmaceutics, 304: 63-71

PINTO, T. J. A.; KANEKO, T. M.; OHARA, M. T. Controle biológico de qualidade de produtos farmacêuticos, correlatos e cosméticos. São Paulo: Atheneu, 2000. 